\title{
1984 y los peligros del totalitarismo
}

\author{
Bárbara MARTíNEZ MARTÍNEZ \\ Universidad Complutense de Madrid \\ barbarma@ucm.es
}

\begin{abstract}
RESUMEN
El objetivo de este estudio es exponer y analizar las posibles consecuencias que sufriría Europa y, más en concreto Reino Unido, en el caso de que los regímenes totalitarios llegaran al poder. Para ello, el estudio se centrará en la novela distópica 1984, escrita en un periodo rodeado de episodios bélicos (Guerra Fría, posguerra...) que muestra algunas de estas consecuencias, entre las que se encuentran el control absoluto de la sociedad, la pérdida de libertades, la censura, el espionaje y el pánico al gobierno del terror.
\end{abstract}

Palabras clave: totalitarismos, distopía, poder, control, deshumanización, terror.

\section{4 and The Dangers of Totalitarianism}

\begin{abstract}
The aim of this study is to show and analyze some possible consequences that Europe and, concretely United Kingdom, could undergo, if totalitarian regimes had access to power. To achieve this objective, the study will be centered on the dystopian novel 1984, written in a period surrounded by war episodes (Cold War, Post-war...), which illustrates some of these consequences such as absolute control of society, loss of freedom, censorship, espionage, and government of terror.
\end{abstract}

Keywords: totalitarianism, dystopia, power, control, dehumanization, terror.

Cuando 1984 comienza a escribirse (1947) y cuando se publica (1949), Europa sigue recuperándose de la situación vivida durante la Segunda Guerra Mundial. Han pasado pocos años desde el fin de aquella guerra monstruosa, iniciada por la Alemania Nazi, que dejó tras de sí episodios tales como el Holocausto o el uso de armas nucleares y que sumieron a Europa en un duro estado de desconcierto, sufrimiento y pobreza del que tardaría en recuperarse, si es que finalmente lo hizo. Una de las consecuencias de esta guerra fue la distinción de dos superpotencias que dividirían al mundo en dos ejes: Estados Unidos (eje capitalista junto con Europa Occidental) y la Unión Soviética (eje comunista junto con Europa Oriental). Asimismo la Segun- 
da Guerra Mundial trajo consigo el miedo a los movimientos totalitarios, y más concretamente fascistas, que hasta ahora solo habían gobernado mediante prácticas terroríficas. Este miedo, que seguía arraigado, se incrementó con el inicio de una guerra entre las dos superpotencias conocida como la Guerra Fría, que comenzó prácticamente al finalizar la Segunda Guerra Mundial, aunque las tensiones entre las potencias venían de antes, y que duraría hasta a disolución de la Unión Soviética.

Reino Unido tuvo también un papel en este conflicto. Hammond afirma que «the involvement of British governments in European security, covert operations, arms talks, propaganda and summitry is now acknowledged» (2011:662). El miedo al comunismo en el Reino Unido por parte del gobierno se palpaba. Los intelectuales de izquierdas apoyaban este movimiento y esto condujo a un despliegue masivo de propaganda antisoviética que se emitía a través de los medios de comunicación y otros métodos como discursos políticos (Hammond 2011: 663). Además el país pasaba también por un periodo de crisis económica que repercutía en la industria de guerra y defensa e incrementaba el miedo a que el enemigo comunista se adentrara en las colonias británicas. Este momento de debilidad le supuso al país perder el «estatus de 'gran poder'» del que una vez habría gozado (Hammond 2011:663). En definitiva, Reino Unido se vio rodeado de un enemigo poderoso que avanzaba por el este y la pérdida de poder proveniente del oeste, lo que le llevó a estar en búsqueda continua de poder. Hammond sugiere que una de las consecuencias más notables de esta situación en Reino Unido fue el miedo al idealismo político (2011:664), además de otras que señala en el siguiente fragmento:

Just as Britain helped to shape the course of global events, so those events shaped aspects of British life, from the Welfare State and the peace movement, through nuclearization, decolonization, unionism, and recession, to Labour's retreat from socialism and the quandaries of post-imperial nationhood (2011:663)

En el lecho de estos acontecimientos, George Orwell se aventuró a escribir una novela distópica, 1984, mediante la cual plasmaría las preocupaciones de la sociedad en que vivía. Orwell escogió como escenario Inglaterra y, en concreto, la ciudad de Londres trasladada al futuro, para especular sobre una posible sociedad dominada por un régimen totalitario y el resultado no fue nada optimista.

La interpretación de la novela fue bastante variada, ya que los críticos no se ponían de acuerdo en los temas sobre los que el texto hacía una reflexión. Así, algunos consideraron que era «a critique of capitalism, totalitarianism, socialism, modern technology, mass advertising, the British Labor Party, English intellectuals, the Welfare State, the Cold War, nuclear weapons, and the isolation and anomie of modern life» (Bergman 1997:174). Las diferentes posibles interpretaciones condujeron a los críticos a reflexionar sobre la orientación política del propio escritor que había sido asociada a la izquierda de la cual ahora parecía alejarse. Así, Orwell fue tachado de adjetivos como «anarchist, a disillusioned socialist, or a conservative reactionary» (Beadle 1975:287). Sin embargo, a pesar de los variados significados de la novela, la mayoría de críticos resumía que era un ataque a la Unión Soviética y el comunismo, debido a la fecha de su publicación que coincidía con el apogeo de 
la Guerra Fría, e incluso al socialismo en general (Beadle 1975:297). Esta exégesis se podría apoyar quizás en la inclusión del escritor como uno de los pocos intelectuales de izquierdas no-marxistas (Beadle 1975:294). No obstante, el pensamiento de Orwell no se alejaba totalmente del Marxismo. Estaba de acuerdo en el ataque al sistema de clases, al imperialismo y defendía, por ejemplo, la igualdad económica, pero estaba en desacuerdo con los valores morales. Además, afirmaba que el régimen de la Unión Soviética no era socialismo o marxismo, sino otra forma, sin valores morales, del sistema capitalista (Beadle 1975: 294; 295). Es posible que está visión antisoviética del escritor condujera a la reducción de su novela a simplemente una crítica al comunismo de la Unión Soviética.

Pese a -o, se podría decir, debido a- esta oposición al marxismo y pese al hecho de que Orwell seguía siendo considerado miembro de los intelectuales de izquierdas, el texto fue utilizado por ambos extremos como propaganda de sus respectivos pensamientos. Por ejemplo, según Hammond, en América el texto se reprodujo masivamente y se utilizó para defender el capitalismo como «la causa del mundo libre» (2011:665). Por el contrario, en la Unión Soviética, 1984 sufrió la censura de las autoridades. Sin embargo, las copias reducidas que circulaban se vendían como una defensa del comunismo y una exaltación a los fallos del capitalismo y el fascismo, aunque los contrarios al régimen dentro de la Unión Soviética también veían en el texto una crítica exclusiva al comunismo soviético (Bergman 1997:177).

Teniendo en cuenta la variedad de interpretaciones y las especulaciones sobre su orientación política, Orwell, en una carta a Mr. Francis A. Henson (miembro de United Auto Workers), escrita en 1949, explicó la intención de su novela:

My recent novel is NOT intended as an attack on Socialism or on the British Labour Party (of which I am a supporter) but as a show-up of the perversions to which a centralized economy is liable and which have already been partly realized in Communism and Fascism. . . I believe also that totalitarian ideas have taken roots in the minds of intellectuals everywhere, and I have tried to draw these ideas out of their logical consequences. . (Beadle 1975:297-98)

En definitiva, la intención de Orwell era mostrar las posibles consecuencias de una sociedad regida por un gobierno totalitario, sin necesidad de nombrar el lado de la balanza al que pertenecía este régimen imaginario que el escritor representó a través del Gran Hermano. Por tanto, la asociación exclusiva del sentido de la novela a la crítica al comunismo o del fascismo sería limitar su significado, ya que lo que se refleja es el peligro de ambos extremos, es decir, de cualquier tipo de totalitarismo.

Para reflejar estos peligros, Orwell, como adelantábamos antes, escoge la distopía, modo de escritura que tuvo bastante auge durante los años de la Guerra Fría (Hammond 2011:664). En su novela retrata una sociedad que ha perdido todo tipo de valores y que vive bajo la atenta mirada del gobierno, sin ningún tipo de libertad. Franja Aérea 1 (Airstrip One) es el futuro nombre de un país, conocido en el presente como Inglaterra, que está sometida a un régimen totalitario representado por el Gran Hermano. Este régimen gobierna basándose en una búsqueda continua de 
poder sin un objetivo -es decir, busca el poder por el poder-, poniendo en práctica un sistema de control absoluto y reduciendo a los individuos a meros objetos con el fin de mantener ese poder.

Para analizar los peligros de este gobierno totalitario, es interesante empezar por el tipo de sociedad en el que el protagonista de la novela, Winston, se desenvuelve. Winston reside en Londres. Esta ciudad no coincide con la ciudad homónima del tiempo de escritura de la novela, ya que Londres en 1984 es la ciudad principal de un nuevo país, Franja Aérea 1 que, a su vez, forma parte de un superestado llamado Oceanía. Además, Winston vive (aunque no lo sabe con certeza) en el año 1984, es decir, en un futuro no muy lejano con respecto al tiempo de publicación o escritura de la novela. A pesar de situarse en el futuro, la ciudad descrita tiene las características propias de una ciudad que ha sido víctima de la guerra. Como Clune comenta, la descripción es similar a la de la propia ciudad de Londres en 1947/1949 que sufría una situación de postguerra (2009:34), lo que proporciona cierto grado de familiaridad al lector y propone que esta situación es posible en cualquier parte del mundo. Además la visión de Winston de esta sociedad está envuelta en un tono pesimista que se deja ver en fragmentos como el siguiente:

Outside, even through the shut window-pane, the world looked cold. Down in the street little eddies of wind were whirling dust and torn paper into spirals, and though the sun was shining and the sky a harsh blue, there seemed to be no colour in anything, except the posters that were plastered everywhere (Orwell 1987:4).

La descripción evoca una imagen de desolación, de ciudad gris, en la que no hay nada vivo excepto el Gran Hermano. Todo lo que rodea al protagonista es un recuerdo continuo de la vigilancia a la que está sometida la población y de que deben jurar lealtad al partido. Unas líneas más adelante, Winston desarrolla la descripción de la ciudad preguntándose si cuando era un niño también era así:

Were there always these vistas of rotting nineteenth-century houses, their sides shored up with baulks of timber, their windows patched with cardboard and their roofs with corrugated iron, their crazy garden walls sagging in all directions? And the bombed sites where the plaster dust swirled in the air and the willowherb straggled over the heaps of rubble; and the places where the bombs had cleared a larger patch and there had sprung up sordid colonies of wooden dwellings like chicken-houses? (Orwell 1987:5)

Lo que se observa en este fragmento es de nuevo una ciudad en ruinas que refleja perfectamente el estado interior de los individuos que la habitan y, en especial, de Winston. Además nos da pistas del desconcierto en el que viven los individuos, ya que una de las tácticas del gobierno para conservar el poder será manipular el pasado y, por tanto, los recuerdos.

Teniendo en cuenta el retrato de la sociedad que ha creado el Gran Hermano -austera, gris, desolada, controlada y reprimida-, la siguiente pregunta a responder es cómo funciona el régimen del Gran Hermano y por qué puede ser considerado un régimen totalitario. Lo primero que debemos hacer para responder a estas preguntas 
es analizar las tácticas del régimen para conservar el poder y señalar a través de qué organismos las imponen.

Lo que se observa a priori es que el régimen del Gran Hermano, el ingsoc (socialismo inglés), se mantiene en el poder a base del control de los individuos que conforman la sociedad, castigando a aquellos que se descontrolen -es decir, gobiernan creando terror-. El partido lleva a cabo la imposición de una realidad ideal que elimina la realidad externa al régimen. El partido deshumaniza a los individuos y los convierte en objetos que pueden ser manipulados y controlados a su antojo. Esta es, pues, la función de los cuatro ministerios entre los que se reparte el poder del Gran Hermano.

El que más nos concierne es el Ministerio de la Verdad, puesto que es donde Winston trabaja, «which concerns itself with news, entertainment, education and the fine arts» (Orwell 1987:6). Para que el partido se mantenga en el poder, lo primero que deben hacer es manipular las mentes, imponerles su verdad y vigilar que no se salgan del orden establecido. Se inculcan a los individuos desde la infancia nuevas normas respecto a prácticamente cualquier aspecto cotidiano como el matrimonio, los placeres, el divorcio, la familia e, incluso, el lenguaje. Por ejemplo, el amor y el sexo por placer van en contra de la ley. El individuo no ha de desarrollar ningún acto que se escape del control del partido. Julia, el personaje con el que Winston se dedica a romper las leyes del partido, le explica lo siguiente:

'When you make love you're using up energy; and afterwards you feel happy and
don't give a damn for anything. They can't bear you to feel like that. They want you
to be bursting with energy all the time. All this marching up and down and cheering
and waving flags is simply sex gone sour. If you're happy inside yourself, why should
you get excited about Big Brother and the Three-Year Plans and the Two Minutes
Hate and all the rest of their bloody rot?' (Orwell 1987:139)

El individuo de Londres debe estar reprimido, no debe dar rienda suelta a sus deseos y placeres y para Julia solo hay un motivo: si eres feliz, no te preocupas por el partido. El gobierno reprime los deseos de sus ciudadanos, de manera que no puedan desarrollar ningún pensamiento individual fuera de los impuestos por la ley. Sin embargo, engaña a los ciudadanos organizando eventos, como la Semana del Odio o los Dos Minutos de Odio, para aparentar que el individuo es libre de odiar -y demostrar ese odio- al enemigo del partido. Se podría decir que aparentando la existencia de odio, se aparenta la existencia de cualquier otro sentimiento, aunque la realidad es que los sentimientos positivos están ocultos y que solamente se puede expresar odio hacia los contrarios al régimen. En todo caso, el amor y el sexo son en esta sociedad actos revolucionarios.

La inexistencia de amor implica que las instituciones como la familia, el matrimonio o el divorcio cambien. El propósito del matrimonio es concebir hijos (el sexo con este propósito se acepta, aunque lo «natural» es tenerlos por inseminación artificial). Para casarse, un comité debe aprobar la unión y, según Winston, si se nota cierta atracción física, la unión no es aprobada. El divorcio no está permitido aunque sí lo está la separación en el caso de parejas sin hijos. Por último, los niños están 
educados en instituciones públicas que les inculquen estos valores y además se les prepara como espías para denunciar a sus padres si escuchan alguna palabra o información en contra del régimen (Orwell 1987:68-9; 140). La sociedad está estructurada de manera que no se pueda escapar de las fauces del partido. Ni siquiera en tu propia casa existe la privacidad, bien sea por tus hijos o bien sea por la presencia de la telepantalla.

La mente de los individuos está continuamente siendo moldeada para que acepte la verdad del partido. Al régimen, por tanto, no le conviene que el individuo tenga la capacidad de desarrollar su propio pensamiento o criterio. Para evitar esto, el gobierno propone, aparte de las medidas anteriores, un nuevo idioma a partir de una modificación de la lengua que existía antes de su llegada al poder: la neolengua ${ }^{1}$ (newspeak). Esta modificación consiste en la eliminación o la alteración de palabras que existían en la viejalengua (oldspeak). Syme, un amigo filólogo de Winston, es el encargado de explicarle el funcionamiento y el propósito de este nuevo idioma. El objetivo es eliminar el «pensamientocrimen» (thoughtcrime), los pensamientos contrarios a las ideas del régimen. Syme explica que llegará un punto en el que el lenguaje esté reducido al mínimo (sin sinónimos, antónimos o connotaciones) y, por tanto, no existirán palabras para expresar o incluso pensar ideas contrarias al régimen. De hecho, la eliminación de palabras provocará que desaparezcan ciertos conceptos. Por ejemplo, si se eliminara «malo» y se usara «bueno» en diferentes grados, para expresar lo que antes era 'malo', tanto el concepto «mal» como el concepto «bien» cambiarían e incluso desaparecerían puesto que tienen una relación dialéctica. Esa nueva lengua favorecerá además la autodisciplina, ya que el individuo no se planteará si existen pensamientos contrarios al régimen (Orwell 1987:546). A pesar de que la neolengua no está implantada aún en el tiempo de Winston, existen ciertas normas con respecto a lenguaje que los ciudadanos deben usar. Por ejemplo, deben dirigirse a los demás como «camaradas» y no deben utilizar palabrotas. El uso de palabrotas se convierte, pues, en otro acto de rebelión.

El proceso de educación en esta sociedad es continuo, puesto que el control absoluto de los pensamientos de los individuos es una tarea ardua y siempre existe el riesgo de que alguno se salga del orden. Por esto, es importante no solo inculcar ciertas normas, sino también hacer creer al individuo que son las únicas y verdaderas normas. De ahí que el gobierno ponga tanto empeño en tareas como la modificación del pasado que ya habíamos mencionado. Los habitantes de Londres en 1984 viven en una realidad ideal creada por y para el partido con la idea de mantenerlo en el poder. Como afirma Cheng, el objetivo del Gran Hermano no es buscar una justificación para su poder y sus métodos, sino eliminar aquello que lo contradice, de manera que la única verdad posible sea la suya (2001:1110-15). No se alza en el poder por sus propios medios, demostrando que lo que hace es lo mejor para la población, sino que lo hace a expensas de coaccionar y engañar a la sociedad sobre la que gobierna.

\footnotetext{
${ }^{1}$ Al final de la novela, Orwell añade un apéndice en el que explica detalladamente el funcionamiento de la neolengua.
} 
Para mantenerse indiscutido, como decíamos, elimina y modifica episodios del pasado. De esto se encarga también el Ministerio de la Verdad y, en concreto, Winston. Él se encarga de cambiar los contenidos de las noticias del pasado para que favorezcan lo que ocurre mientras gobierna el Gran Hermano, con la posterior destrucción de la copia original. Puede «vaporizar» una persona, si ha sido eliminada por el régimen y se tiene que hacer ver que nunca ha existido. Incluso a veces debe modificar las propias noticias del partido, por ejemplo, cuando cambia el enemigo de guerra para enseñar que el nuevo enemigo ha sido el enemigo siempre. Además, no solo las noticias son modificadas o eliminadas, también eliminan el arte y cualquier forma de entretenimiento. Como los nazis hicieron, en Oceanía también hubo una destrucción masiva de libros. Los edificios fueron descontextualizados: si era enorme y relativamente nuevo, el partido lo construyó, sino fue construido en una etapa previa al capitalismo. Asimismo, «statues, inscriptions, memorial stones, the names of streets -anything that might throw light upon the past had been systematically altered» (Orwell 1987:102). Las novelas, poemas, películas o canciones son creados ahora a partir de máquinas - contribuyendo a la deshumanización de la sociedad $-\mathrm{y}$ todas con el objetivo de ensalzar al partido.

No hay manera, por tanto, de conocer el pasado, ya que todo lo relacionado con él se elimina o altera. La conclusión de Winston es «... if all other accepted the lie which the Party imposed -if all records told the same tale- then the lie passed into history and became truth» (Orwell 1987:37). Entonces, si el partido cuenta una información y no existe ninguna otra información que la desmienta, automáticamente se convierte en verdad. Como sugiere O'Brien, el pasado no existe en ningún espacio concreto, solo en documentos y en la memoria. Si el gobierno es capaz de controlar estos dos aspectos controla el pasado (Orwell 1987:260). La modificación del pasado permite al gobierno controlar el presente y conduce a los ciudadanos a estar en un estado de desconcierto que provoca que no tengan más remedio que creerse lo que ven, lo que les imponen en su presente, puesto que es su única realidad.

El Ministerio de la Verdad se encarga, por tanto, de que el individuo no se salga del orden mediante la educación dirigida, la represión de sus instintos, la modificación de su historia y la recreación de una realidad ideal que no tiene más remedio que aceptar. Además, existe el Ministerio de la Paz, «which concerned itself with war» (Orwell 1987:6) y mantiene al Estado en guerra continua. Julia comenta a Winston que el objetivo de esto es «keep people frightened» (Orwell 1987:160). Es decir, que la guerra podría ser mentira pero afirmar que existe es una manera de que el partido instaure su realidad, de mostrar que el partido no es el enemigo, ya que está salvando a la población, y de enseñar a la vez que es un enemigo poderoso. De esa manera, no habría oposición.

E1 Ministerio de la Abundancia «was responsible for economic affairs» (Orwell 1987:6). Este ministerio es el responsable de lo que al principio veíamos como una sociedad austera, desolada y gris. La sociedad es víctima de un sistema de racionamiento que les permite consumir lo necesario; no pueden adquirir ningún producto que no sea práctico. La moneda de cambio son «cupones» con los cuales tienen que apañarse todo el año y las raciones de comida van variando, normalmente a la baja, aunque al pueblo se le hace creer que van aumentando. Aunque toda la sociedad 
sigue este sistema, hay diferentes restricciones según tu puesto laboral. Así, un miembro del Partido Externo, no podrá acceder a los mismos sitios o no podrá consumir lo mismo que un proletario. Cuanto más alta sea la categoría, mayor será la restricción.

Por su parte, el Ministerio del Amor «maintained law and order» (Orwell 1987:6). Este ministerio es el que se encarga de todos aquellos que se opongan o tengan intención de oponerse al régimen. Igual que eliminan el pasado para favorecer su ideología o eliminan las pruebas de que las raciones están siendo reducidas, así hacen con los contrarios a sus ideas (Cheng 2001:1111). La Policía del Pensamiento (Thoughtpolice) es la responsable de identificar y atrapar a todos aquellos que, aunque no han cometido ningún delito contra el partido, podrían hacerlo en un futuro. Aquellos que son arrestados, como sucede con el protagonista, son sometidos a una reeducación (un lavado de cerebro a base de torturas), ya que, en palabras de Winston, el gobierno no puede permitirse ni la más mínima desviación. Después de someterlos al lavado de cerebro, son matados y eliminados de la historia. Winston es sometido a este método y finalmente se puede observar cómo, a pesar de su lucha, acaba creyendo firmemente en el partido.

El proletariado, observamos, es el único sector que se salva de muchas de las restricciones que impone el partido y además apenas está vigilado por la Policía del Pensamiento, ya que consideran que no son un peligro. Siguiendo esto, Winston deduce que la esperanza de que el régimen caiga reside en el proletariado $(85 \%$ de la población). Como sugería Julia, ellos son los únicos capaces de satisfacer sus deseos y placeres, incluso se crean modos de entretenimiento dedicados a ellos, por tanto, son los únicos que están felices. Sin embargo, siguiendo la teoría de Julia, al estar felices se olvidan del partido, olvidan a lo que están sometidos, no son conscientes de la opresión y, por ende, nunca se rebelarán. O'Brien afirma que debido a que el proletario no tiene con qué comparar su vida, no es consciente de su estado de opresión (Orwell 1987:216).

La sociedad de Oceanía y, concretamente, de Londres está dominada por el gobierno del terror; está sometida a una educación ortodoxa, a restricciones físicas y mentales y a una vigilancia obsesiva, y si se salen de la norma son aniquilados. Pero existen otros rasgos que nos dirigen a pensar que este gobierno es un régimen totalitario; por ejemplo, el culto al líder, la divinización del partido y la demonización de los enemigos -reforzado mediante rituales, como los Dos Minutos de Odio, o propaganda como los posters y panfletos que rodean la ciudad...- y la idea de que con su ejercicio de poder está creando la sociedad perfecta, ideal, utópica. Esto es lo que Eloísa Suárez denomina «religión política», término que asocia al estalinismo y al nacionalsocialismo (2008:166-167). Explica que este tipo de regímenes «utili$\mathrm{za}[\mathrm{n}]$ estructuras religiosas y simbolismos para propósitos político-ideológicos. Además suele[n] buscar el desplazamiento y la erradicación de las creencias religiosas tradicionales» (Suárez 2008:166). No estaría de más añadir que el régimen del Gran Hermano no solo acaba con las creencias religiosas, si no que acaba con cualquier creencia moral, ya que su gobierno se basa en conseguir y mantener el poder sin ningún tipo de escrúpulos. 
Las posibles consecuencias de los regímenes totalitarios a las que llega Orwell en el texto están impregnadas de un tono pesimista. Lo que propone 1984 es la descripción de una ciudad en ruinas, una sociedad permanentemente vigilada, sin espacios privados (ni siquiera en sus propias casas), controlada por el gobierno mediante medios tan inmorales como el lavado de cerebro; el sometimiento a los ciudadanos a una educación estricta; la reducción de los individuos a objetos manipulables y la consecuente pérdida de libertades. Los instintos son reprimidos y los placeres, prohibidos. El pensamiento individual ha de ser evitado. Incluso llegan a crear una nueva lengua con el objetivo de eliminar los pensamientos contrarios al partido. Los ciudadanos viven permanentemente en alerta para no ser descubiertos con un gesto o una postura sospechosa porque incluso sus hijos les pueden delatar. Los peligros del totalitarismo, que refleja 1984 y que podría ser trasladado a cualquier país o ciudad europeos, son, por tanto, el gobierno del terror y la consecuente ruina económica, política, social y moral que implica la deshumanización de los individuos, la falta de pensamiento individual y la experiencia de una realidad manipulada.

\section{Bibliografía:}

BeadLE, Gordon B. (1975): «George Orwell and the Victorian Radical Tradition». Albion: A Quarterly Journal Concerned with British Studies 7, 4: 287-299.

Bergman, Jay (1997): «Reading Fiction to Understand the Soviet Union: Soviet Dissidents on Orwell's 1984». History of European Ideas 23, 5-6: 173-192.

CHENG, Sinkwan (2001): «Ressentiment, the Superego and Totalitarianism: George Orwell's 1984». Cardozo Law Review 24, 3: 1099-1130.

Clune, Michael (2009): «Orwell and the Obvious». Representations 107, 1: 30-55. Hammond, Andrew (2011): "The Twilight of Utopia': British Dystopian Fiction and the Cold War». The Modern Language Review 106, 3: 662-681.

ORWELL, George (1987): 1984. Londres: Penguin Books.

SuÁrez López-ZuriagA, Eloísa (2008): «Estalinismo y religión política: entre la ficción y los acontecimientos históricos». Bajo palabra 3: 165- 172. 